\title{
NOTIZEN ZUR SPÄTEN GESCHICHTE DES BARȘAUMŌ-KLOSTERS
}

\author{
HUBERT KAUFHOLD \\ LEOPOLD-WENGER-INSTITUT FÜR RECHTSGESCHICHTE \\ DER UNIVERSITÄT MÜNCHEN
}

\section{ZUSAMMENFASSUNG}

Zur Zeit Michaels des Syrers, aber auch darüber hinaus, war das Kloster des bl. Barsaumō Residenz der syrischen Patriarchen. Nach der grundlegenden Darstellung von Ernst Honigmann (Le couvent de Barșaumā, 1954) endete die Geschichte des Klosters gegen Ende des 13. Jh. Aber schon Patriarch Aphram Barsaum wies in seiner Geschichte der syrischen Literatur (2. Aufl. 1956) darauf hin, wenn auch obne nähere Angaben, daß das Kloster bis zum Ende des 17. Jh. bestand. Im folgenden Beitrag werden historische Notizen über das Kloster bis 1676 n. Chr. zusammengestellt, die hauptsächlich aus Kolophonen von Handschriften stammen.

\section{I}

[1] Nach seinem Tod vor 800 Jahren, am 7. November 1199, wurde Patriarch Michael der Syrer ("der Ältere") im Barșaumō-Kloster begraben. Barhebraeus berichtet in seiner Kirchengeschichte, daß Michael in der "neuen Kirche," die er von 1180 bis 1193 im Kloster hatte erbauen lassen, beigesetzt wurde, "in einem Grab vor dem nördlichen Altar, das er bereits zu seinen Lebzeiten 
eingerichtet hatte." ${ }^{1}$ Ernst Honigmann ${ }^{2}$ hat mit guten Gründen das Kloster mit den Ruinen von Borsun Kalesi identifiziert, die sich auf einem ungefähr $1600 \mathrm{~m}$ hohen Berg zwischen Malatya und Adiaman in der Südosttürkei — unweit des bekannten Nimrud Dag - befinden. Dort sind Reste einer Kirche mit drei Apsiden erhalten. Wegen der Zerstörungen des Klosters und des an der betreffenden Stelle aufgehäuften Schutts war bisher nicht feststellbar, ob sich dort das Grab befindet. ${ }^{3}$ Ausgrabungen wurden noch nicht durchgeführt.

Die Anfänge des Barșaumō-Klosters lassen sich bis zum Ende des 8. Jh. zurückverfolgen. Es spielte zunächst wohl keine besondere Rolle, gewann aber im 11. und 12. Jh. größere Bedeutung. ${ }^{4}$ Vor allem Michael, der zunächst Abt des Klosters war, ließ mehrere größere Baumaßnahmen durchführen. Nach seiner Wahl zum Patriarchen 1166 hielt er sich — wie schon einige seiner

1 Joannes Baptista Abbeloos et Thomas Josephus Lamy, Gregorii Barbebraei chronicon ecclesiasticum, tomus I (Leuven, 1872) 605/606. In der bis zum Jahre 1234 reichenden anonymen Chronik ist der Teil, in dem der Bericht über Michaels Tod gestanden haben dürfte, nicht erhalten, vgl. Jean-Baptiste Chabot (Hrsg.), Anonymi auctoris chronicon ad A. C. 1234 pertinens, II (Leuven, 1916) 335, bzw. die Übersetzung von Albert Abouna und Jean Maurice Fiey (Leuven, 1974) 250 (= CSCO 82, 354).

2 Ernst Honigmann, Le couvent de Barșaumà et le patriarcat jacobite d'Antioche et de Syrie (CSCO 146; Leuven, 1954).

3 Hansgerd Hellenkemper, Burgen der Kreuzritterzeit in der Grafschaft Edessa und im Königreich Kleinarmenien (Bonn, 1976). Zum BarșaumōKloster: insbesondere S. 99-103 (hier 102). Abbildungen: Tafeln 18, 19. Vgl. auch M. Thierry, "Monuments chrétiens inédits de HauteMésopotamie," Syria 70 (1993): 179-204 (192-5: III. Le couvent de Barsauma [mit einem Photo und einem Grundriß der Kirche]). Weitere Photos von André Maricq und Friedrich Karl Dörner sind bei Honigmann, Le couvent de Barșaumā, bzw. Peter Kawerau, Die jakobitische Kirche im Zeitalter der syrischen Renaissance (Berlin, 1960), abgedruckt.

4 Es hatte seine eigene liturgische Ordnung, die der Metropolit Dionysios von Melitene Anfang des 11. Jh. festgelegt hatte (Jean-Baptiste Chabot, Chronique de Michel le Syrien, tome III (Paris, 1905) 190). Die Hs. Vat. Syr. 51 nimmt Bezug auf den Ritus des Klosters des Mōr Barṣaumō (Stephus Evodius und Joseph Simonius Assemani, Bibliothecae Apostolicae Vaticanae codicum manuscriptorum catalogus, partis primae tomus secundus (Rom, 1758) 322), die Hs. Brit. Libr. 14,716 enthält Hymnen nach der "Ordnung des Klosters des Mōr Barșaumō" (William Wright, Catalogue of Syriac Manuscripts in the British Museum, part 1 (London, 1870) 375a). 
Vorgänger - des öfteren im Kloster auf, so daß man es als Patriarchatsresidenz bezeichnen kann. Von 1074 bis 1283 fanden dort mehrere Synoden statt. ${ }^{5}$ Auch Michaels Nachfolger im 13. Jh. lebten teilweise in diesem Kloster. Nach dessen Zerstörung hielten sie sich zunächst in Sis im armenischen Königreich Kilikien auf, bevor das Hananyā-Kloster bei Mardin (Dair az-Za farān) der bevorzugte Aufenthaltsort der Patriarchen wurde. Die insbesondere aus den Chroniken bekannten Daten hat Honigmann zusammengestellt, so daß darauf verwiesen werden kann. ${ }^{6} 1285$ wurde das Kloster durch ein Erdbeben erneut zerstört. Als letztes Ereignis erwähnt Honigmann eine Plünderung des Klosters durch die Kurden im Jahre 1293 oder wenig später. ${ }^{7}$

Die Geschichte des Klosters war damit jedoch nicht beendet, wie es nach der Darstellung Honigmanns scheinen könnte. ${ }^{8}$ Der syrisch-orthodoxe Patriarch Ignatius Aphram Barsaum (1887 bis 1957) hat - wie so oft - recht, wenn er in seiner Geschichte der syrischen Literatur darauf hinweist, daß das Kloster bis zum Ende des 17. Jh. bestand. ${ }^{9}$ Er macht jedoch keine näheren Angaben. Im folgenden sollen deshalb einige Lesefrüchte vor allem aus syrischen Handschriften zusammengestellt werden, die ein paar Schlaglichter auf die spätere Geschichte des Klosters werfen. Zu seinem alten Glanz hat es offenkundig nie wieder zurückgefunden. 78-92.

${ }^{5}$ Vgl. etwa Joseph Mounayer, Les synodes syriens Jacobites (Beirut, 1964)

${ }^{6}$ Le cowvent de Barșaumä, vor allem S. 47-76. Zu ergänzen sind nur einige Ereignisse, die in der bis zum Jahre 1234 A. D. reichenden Chronik berichtet werden; auf einige wird unten eingegangen.

${ }^{7} \mathrm{AaO} 50 f ., 75$.

${ }^{8}$ So z. B. - unter Berufung auf Honigmann und Hellenkemper, Burgen der Kreuəritterzeit 103: "1285 zerstört ein Erdbeben ein Großteil der Klosterbauten und wohl 1293/94 ist die Stätte von einfallenden Kurden gebrandschatzt worden und in der Folge verfallen." Auch Joseph Simonius Assemani, Bibliotheca Orientalis Clementino-Vaticana, Band 2 (Rom, 1721) S. (97) des unpaginierten Teils macht keine weiteren Angaben.

${ }_{9}$ Histoire des sciences et de la litteratur syriaque [arabisch], 2. Auflage, Aleppo 1956 (mehrere Nachdrucke), 509. Syrische Übersetzung von Ph. Y. Dolabani: Ktōbō da-brullè bdìrè (Qāmišlī, 1967) 633. Auf diese Angabe stützt sich offenbar Jean-Maurice Fiex, Pour un Oriens Christianus Novus (Beirut, 1993) 176: "Le couvent exista jusqu'au milieu du XVII" siècle." 


\section{II}

Die mir bekannten Nachrichten über das Kloster sind leider nur sehr dürftig und lassen auch nicht annähernd eine zusammenhängende Darstellung seiner weiteren Geschichte zu. Sie setzen erst wieder in der zweiten Hälfte des 15. Jh. ein. Dies deutet darauf hin, daß es fast zwei Jahrhunderte tatsächlich verfallen und unbewohnt war. Das Gebiet, in dem es liegt, gehörte seit dem Schisma 1292/3 zur kilikischen Linie der Patriarchen, die später in Damaskus residierte. Dieses kilikische Patriarchat hatte wohl bald keine große Bedeutung mehr und erlosch 1444/5. Wegen des allgemeinen Niedergangs konnte das Barșaumō-Kloster zunächst wohl nicht wiederbelebt werden. ${ }^{10}$ Es gab aber in der Gegend damals zweifellos syrische Bevölkerung. Seit dem Ende des 14. Jh. ist eine lange Reihe von syrisch-orthodoxen Bischöfen von Gargar belegt. ${ }^{11}$ Gargar (heute Gerger), ${ }^{12}$ in dem es eine Kirche der Muttergottes und des hl. Barșaumō gab, ${ }^{13}$ liegt unweit des Barșaumō-Klosters in der Nähe des Euphrat. In dem Gebiet bestanden zwei andere, in unserem Zeitraum sicher bedeutendere syrische Klöster, nämlich das "Kloster der Stufen" (Dairō d-seblōtô), auch: Kloster des Mōr Abhai, am Euphrat, ${ }^{14}$ und das Kloster des Abū Gālib (oder: der Muttergottes und des Zachäus [Zakai]) bei Wank. ${ }^{15}$ Im Kolophon der Hs. Jerusalem 62 werden noch zwei weitere Klöster genannt: (das Klosters des Mōr Abḥai liege) “im Gebiet von Gargar, am nördlichen Abhang (des Flußufers?) in der Nähe des Klosters der Barfüßer (šamitāyè ), das Pesqīn genannt wird, und gegenüber dem Kloster des Mōr Šabtai, das Šìrā benannt ist.”16

10 Yohannan Dolabani, Die Patriarchen der syrisch-orthodoxen Kirche von Antiochien [syrisch], Holland (Glane/Losser, 1990) 181 Z. 7 von unten.

${ }^{11}$ Fiey, Pour un Oriens Christianus Novus, 199-201 (die Liste läßt sich noch erweitern).

12 Honigmann, Le Couvent de Barșaumā, 123 (Nr. 40); Hellenkemper, Burgen der Kreusritterzeit, 79-83.

13 W. F. Ainsworth, Travels and Researches in Asia Minor, Mesopotamia, Chaldea and Armenia (London, 1842) 277, und K. Humann, O. Puchstein, Reisen in Kleinasien und Syrien (Berlin, 1890) 354, berichten nur von einer armenischen Kirche mit einem Priester.

${ }^{14}$ Honigmann, Le couvent de Barșaumā, 81f. (No. 15).

${ }^{15}$ Ebd., 80 (No. 10).

16 Filoksinos Yohanna Dolabany, Catalogue of Syriac Manuscripts in St. Mark's Monastery. Ed. by Gregorios Yohanna Ibrahim (Aleppo, 1994) 
Wahrscheinlich soll damit nur die örtliche Lage beschrieben werden. In sonstigen Quellen werden diese älteren Klöster nicht erwähnt. Wahrscheinlich waren sie nicht mehr bewohnt; das bestätigt eine Handschrift aus dem Jahre 1599 ausdrücklich (s. unten [21]).

Wir wissen darüber hinaus von einigen Ortschaften im Gebiet von Gargar, in denen es auch später noch syrische Kirchen gab: 'WYWS (Aussprache: Āwios?) (Barșaumō-Kirche), Halhal (?, HLHL) (Māmā-Kirche), Karmō d-Dakyō (?; KRM’ DDKY’17) (Barṣaumō-Kirche), Mağzūnīr (?; MGZWNYR bzw. MGZZWNYR ${ }^{18}$ oder MaNGZNR ${ }^{19}$ ) in der Nähe des Klosters des Mōr Abhai (Abhai -Kirche), Tabsiyas ${ }^{20}$ (Barșaumō-Kirche, Muttergotteskirche), Urb̄̄šs ${ }^{21}$ (Georgskirche) und Wank ${ }^{22}$ (Muttergotteskirche). Wir kennen ferner aus Handschriftenvermerken viele Personen aus den genannten Orten namentlich. Dabei treffen wir öfter auf armenische Namensformen. Das weist, ebenso wie der Ortsname "Wank" (armenisch: "Kloster") darauf hin, daß wir uns in einer Gegend befinden, in der es zahlreiche Armenier gab.

190. Zur Lage des Klosters vgl. die Beschreibung von Henri Pognon, Inscriptions sémitiques de la Syrie, de la Mésopotamie et de la région de Mossoul (Paris, 1907) 117f. Zu dem Kloster der Barfüßer und dem des Mōr Šabtai vgl. Honigmann, Le couvent de Barșaumaa, 59, 60f., 82 (Nr. 16).

${ }^{17}$ Hs. Dam. Orth. 5/16, Ordinationsliste; Hs. Scharfeh 111 (Behnam Sony, Le Catalogue des manuscrits du patriarcat au couvent de Charfet - Liban [arabisch] (Beirut, 1993) 285b [Nr. 780]: KRMW DHYW. Von der Schrift her ähnelt der Name einer Ortsbezeichnung nahe beim BarsaumōKloster: Karmō (d-metqrē) d-Pìlō "Weinberg des Elefanten," s. Chabot, Chronique de Michel, III 287b (IV 644); Abbeloos-Lamy, Gregorii Barbebraei cbronicon, I 507.

18 Filoksinos Yohanna Dolabany, Catalogue of Syriac Manuscripts, 68 bzw. Sony, Catalogue ... de Charfet, 13 (Nr. 2), 285b (Nr. 780).

${ }^{19}$ Ms. Mardin Orth. 326.

${ }^{20}$ Vgl. Honigmann, Le cowvent de Barsaumā, 80 (Nr. 10). In der noch zu nennenden Hs. Aleppo Orth. 4 wird der Ort wie folgt teilweise vokalisiert: Tbseya's, in der Hs. Aleppo 15: Tbsiyas. Honigmann, Le couvent de Barșaumā, 80, 171, meint, er sei identisch mit "Taraksu," einem Ort, der auf der zweiten Karte Honigmanns eingezeichnet ist.

${ }^{21}$ Honigmann, Le couvent de Barșaumä, 82 (No. 15), 171.

${ }^{22}$ Cf. ebd., 80 (No. 10). 
Nun zu den einzelnen Belegen.

a. Die Hs. Vat. Syr. 564 wurde 1463 im Kloster des Mōr Abhai geschrieben. Im Kolophon gibt der Schreiber an, es liege in der Nähe des Klosters des Barșaumō. ${ }^{23}$ Hieraus kann nicht unbedingt der Schluß gezogen werden, daß letzteres bewohnt war. Möglich ist, daß der Kopist durch die Erwähnung des berühmten Barșaumō-Klosters nur die örtliche Lage bezeichnen wollte.

b. Mein erster sicherer Beleg für die Spätzeit des BarșaumōKlosters ist jedoch nur sechs Jahre jünger und stammt von 1781 A. Gr. (1469/70 A. D.). In diesem Jahr entstand eine Handschrift mit der Geschichte des Apostels Thomas und anderem, geschrieben im Barșaumō-Kloster. ${ }^{24}$ Näheres erfahren wir nicht.

c. Nach dem Kolophon eines 1549 im Kloster Surb Karapet geschriebenen armenischen Tetraevangeliums, das sich in Lille befindet, gaben die Besteller es "zur Erinnerung an die Tür des hl. Barșaumō” (jišatake $i$ durn surb Parsoma). ${ }^{25}$ Anhaltspunkte dafür, daß das syrische Barșaumō-Kloster gemeint ist, sind nicht erkennbar, ein Bezug zu unserem Gebiet fehlt völlig. Für Michel Thierrys Überlegung, die Armenier könnten sich des Klosters bemächtigt haben, ${ }^{26}$ gibt es jedenfalls keinen Grund.

d. In der bisher nicht katalogisierten Hs. Aleppo Orth. 116, die ein Marienleben enthält, steht am unteren Rand einer Seite ein kurzer Vermerk aus dem Jahre 1880 A. Gr. (= 1568/9 A. D.). Darin bittet der Schreiber um ein Gebet für Rabban Malkē, den Abt des Klosters des Mōr Abhai, und für sieben weitere Mönche. ${ }^{27}$ Er fordert dann auf, "für Rabban Hananyā (den Abt?) von Mōr

23 Arn. van Lantschoot, Inventaire des manuscrits syriaques des Fonds Vatican (490-631) Barberini Oriental et Neofiti (Vatikanstadt, 1965) 89.

${ }^{24}$ Hs. Diyarbakir, Başaranlar 41, s. Arthur Vööbus, Handschriftliche Überlieferung der Mèmrè-Dichtung des Ja'qob von Serug, III (CSCO 421; Löwen, 1980) 49. Eine Abschrift davon ist die Hs. Damaskus Orth. 9/15, in deren Beschreibung sich jedoch kein Hinweis auf das Barșaumō-Kloster findet.

25 Frédéric Macler, Rapport sur une mission scientifique en Belgique, Hollande, Danemark et Suède (Paris, 1924) 61, 63.

${ }^{26}$ Monuments chrétiens, 193.

27 Rabban Simeon, Rabban Johannes, Rabban Barșaumā, another Rabban Barșaumā, Rabban Theodoros, Rabban 'Abdallah and Rabban Pilatos. 
Barșaumō (l-Mōr Barșaumō) und Rabban Yū'annīs" zu beten. Da er anscheinend alle Mönche aufzählt, waren die beiden letztgenannten wohl die einzigen Insassen des Barșaumō-Klosters.

e. Etwa zwei Jahre später verkaufte der Priestermönch Slïbō eine von ihm kopierte andere Handschrift mit dem Marienleben (Aleppo Orth. 120) an den Priestermönch Abhai, Sohn des Maqdisī (Jerusalempilgers) Badrān, aus Gargar. Der sprachlich nicht einwandfreie Kolophon fährt fort: 'tktb l-ktb' hn' dyr' d-mry d-mrn mry Brsswm' qlyt' 'ly' 'I mirt' rb' 'l qbrh d-mry Brșwm' 'l k'p'. slw. b-(s)nt 'lp wtmn' m" w-tmnyn w-tryn d-ywny' 'l mirt' d-mry Brswm' ' k'p'. Gemeint ist wohl: "Geschrieben wurde dieses Buch im (?) Kloster des Mōr Barșaumō, in der oberen Zelle bei (über?) der großen Höhle bei (über?) dem Grab des Mōr Barșaumō auf dem Felsen. Betet! [Weiter quer am Rand:] Im Jahr 1882 A. Gr. [1570/1 A. D.] bei (über?) der Höhle des Mōr Barșaumō auf dem Felsen.”

Leider wissen wir nicht genau, wo sich das Grab des Namenspatrons, des berühmten Mönches Barșaumō aus dem 5. Jh. befindet. Begraben wurde er nach der syrischen Vita offenbar in seinem Kloster. ${ }^{28}$ Im Barșaumō-Kloster auf dem Berg verehrte man später nur eine Reliquie, nämlich die rechte Hand. Honigmann nimmt an, daß sich das Kloster, in dem der Heilige lebte, zwar in der näheren Umgebung, aber am Fuße des Berges befand. ${ }^{29}$ In der Vita heißt es nämlich: "Il demeurait l'hiver dans la caverne et l'été ... il allait avec ses disciples sur une montagne eloignée d'environ 25 milles de toute habitation ..." 30 In der Vita wird auch berichtet, daß sich das alte Kloster in einer Höhle befand, die von einem überhängenden Felsen gebildet wurde. ${ }^{31}$ Möglicherweise wohnte also der Kopist der Hs. Aleppo 120, der ebenfalls von einer Höhle und einem Felsen spricht, nicht im späteren Kloster auf dem Berg, sondern beim ursprünglichen Kloster, vielleicht als Einsiedler. Offenbar war damals die Lage des Grabes noch bekannt. Es fällt allerdings auf, daß sonst — etwa in der Chronik Michaels des

${ }^{28}$ François Nau, "Résumé des monographies syriaques: Barsauma...," Revue de l'Orient Chrétien 18 (1913): 270-6, 379-89; 19 (1914): 113-34, 278-89 (hier: Band 19, 284-6).

${ }^{29}$ Le couvent de Barșaumā, 25.

30 Nau, "Résumé des monographies syriaques," 18 (1913): 380. Vgl. auch Honigmann, Le couvent de Barșaumā, 24.

31 Arthur Vööbus, History of Asceticism in the Syrian Orient, volume 2 (Leuven, 1960) 199f. 
Syrers - davon keine Rede ist. Eine Ausnahme macht allenfalls die bis zum Jahre 1234 reichende Chronik, wonach Michael auf der Synode von 1193 im Barṣaumō-Kloster eine Ansprache hielt und am Schluß die Formulierung verwendete “... durch die Gebete der Muttergottes und des heiligen Mōr Barṣaumō, vor dessen Reliquien (Grab?) wir versammelt sind (da-qdōm škinteh etkanašnan)." 32 Wahrscheinlich galten sowohl die Höhle des Heiligen wie das Kloster auf dem Berg als Barṣaumō-Kloster. Daß es zwei Örtlichkeiten dicht beieinander gab, die als Kloster des Barșaumō bezeichnet wurden, ist gut möglich. Das mittelalterliche Kloster lag zweifellos auf einem hohen Berg und war wie eine Festung gebaut. Michael der Syrer berichtet über die Plünderung des Klosters durch Josselin im Jahre 1148 A. D. und dessen Brief an den Emir von Melitene, in dem es heißt: "Ich aber habe das Kloster des Mōr Barșaumō eingenommen, das eine Festung ist, die höher ist als viele, wie ein Adler über den Vögeln." 33 Bei seiner ausführlichen Schilderung des Baus der Wasserleitung für das Kloster erwähnt er die Skepsis der Mönche, "die nicht glaubten, daß man jemals eine Leitung auf dem Gipfel eines Berges wie diesem errichten könne, der voller Steine und Felsen ist." 341066 wurden einige armenische Räuber die Felsen "herabgestürzt" (šdau b-kiifö); ihre Spießgesellen waren "unten" (l-taht) geblieben. ${ }^{35}$ In der Regel wird für den Weg ins Kloster der Ausdruck "hinaufsteigen" (sleq) verwendet. In der bis zum Jahr 1234 A. D. reichenden Chronik finden sich aber auch Hinweise auf ein Barșaumō-Kloster am Fuß eines Berges, offenbar ganz in der Nähe: 1163 war der Winter hart und wegen der Menge des Schnees stieg der Fluß so stark an, daß die Mühle des Klosters überschwemmt wurde; ${ }^{36}$ dieser Fluß kann wohl nur der Kahta Çay

32 Chronicon anonymum ad annum Christi 1234 pertinens, II (= CSCO 82, 354) 317 (Text) bzw. 237 (Übersetzung: “devant le tombeau duquel nous sommes réunis"). Michael selbst berichtet über die Synode in seiner Chronik nur ganz kurz (Chabot, Chronique de Michel, III 387).

${ }_{33}$ Chabot, Chronique de Michel, III 287a (IV 644).

${ }^{34}$ Ebd., 322.

${ }^{35}$ Ebd., 163f. (IV).

${ }^{36}$ Chronicon anonymum ad annum Christi 1234 pertinens, II, 159 (text), 120 (Übersetzung). 
weit unterhalb des Klosters auf dem Berg gewesen sein. ${ }^{37} \mathrm{Im}$ Februar 1206 verwüstete ein Brand die Gebäude des Klosters, ein Jahr später zerstörte ein Erdbeben die neuaufgebauten Bauwerke. Im Winter 1207/8 gab es dann starken Regen, der "zu einem Strom und einem großen Sturzbach wurde, und er stieß mit Heftigkeit gegen das Kloster und zerstörte alles, was er vorfand." 38 Es ist nicht leicht vorstellbar, wie ein Sturzbach auf der Spitze eines Berges solche Schäden verursacht haben sollte, auch wenn das Kloster auf einem Sattel 60 Meter unterhalb des Gipfels lag. ${ }^{39} 1207$ starb Patriarch Athanasios im Barșaumō-Kloster und wurde "in der alten unteren Kirche" (b-idtō taḅtōytō atīgtō) bestattet. ${ }^{40} \mathrm{Da}$ im Kloster auf dem Berg nur die "Neue Kirche" den Brand von 1183 überstanden hatte, müssen wir die "alte Kirche" vermutlich anderswo suchen. Da sie als "die untere" bezeichnet wird, stand sie vielleicht am Fuß des Berges, im alten Kloster des Barșaumō. Möglicherweise bezieht sich auch die Erneuerung der "Wasserquelle des Klosters" (ainō d-mayō ... d-umrô), die Michael der Syrer 1173 A. D. vornahm, ${ }^{41}$ auf ein tiefer gelegenes Kloster, weil — wie Hellenkemper schreibt — "diese Nachricht ... nicht ganz verständlich [ist], denn sie setzt eine natürliche Quelle im Felsgrat voraus, was nach den heutigen topographischen Bedingungen [des Klosters auf dem Berg] wenig wahrscheinlich erscheint." 42 Genauere Feststellungen lassen sich wohl nur an Ort und Stelle und durch Ausgrabungen treffen.

Die Höhle, die auf das alte Kloster des Barșaumō hinweist, wird noch in Handschriften aus den Jahren 1599 und 1622 erwähnt (s. unten [21] und [22]). Bei allen Belegen wäre also zu bedenken,

37 Honigmann, Le couvent de Barsaumā, 39; Hellenkemper, Burgen der Kreu₹ritterzeit, 99; Thierry, Monuments chrétiens, 192. Natürlich könnte die Wassermühle auch getrennt vom Kloster unten am Fluß gelegen haben.

38 Chronicon anonymum ad annum Christi 1234 pertinens, II 346 (Text), 257f. (Übersetzung).

39 So Humann-Puchstein, Reisen in Kleinasien, 206 (abgedruckt bei Honigmann, Le couvent de Barșaumā, 3f.; Thierry, Monuments chrétiens, 193.

40 Ebenda 349 (Text), 260 (Übersetzung). In der Chronik des Barhebraeus ist nicht vermerkt, wo der Patriarch bestattet wurde, s. Abbeloos-Lamy, Chronicon, I 615 (wo die Jahreszahl fälschlich mit "Chr. 1107" statt 1207 angegeben ist.

${ }^{41}$ Chabot, Chronique de Michel, III 350 (IV 703).

42 Burgen der Kreusritterzeit, 101. 
ob wirklich das Kloster auf dem Berg gemeint ist. Für den Aufstieg vom unten gelegenen Dorf Peres zum Kloster auf dem Berg benötigte Thierry übrigens eine Stunde. ${ }^{43}$

f. 1574 A. D. beendete ein unbekannter Schreiber die Hs. Istanbul, Mart Maryam 7. Die Handschrift gehörte früher zur Sammlung des Fehim Bei in Istanbul. Aus dieser Zeit stammt die genaueste Beschreibung, in der es am Schluß heißt: "Beendet am 17. des Monats August im Jahre 1885 A. Gr. im berühmten Kloster (b-umrō tabibōo) des Mōr Barșaumō." 44 Vööbus kannte die Handschrift ebenfalls und gab an einer Stelle an, sie sei "geschrieben im Kloster von Mār Barsaumā in Melitene." 45 Wenn überhaupt, wird in der Handschrift allenfalls etwas stehen wie "im Gebiet von Melitene;" wahrscheinlich jedoch stammt die (unzutreffende) Ergänzung von Vööbus.

g. Etwa zweieinhalb Jahre später entstand die Hs. Scharfeh 5/3, ein Bēt Gazō. Laut einem Vermerk wurde ein Teil von einem Rabban Johannes im Dezember des Jahres 1888 A. Gr. beendet, also 1576 A. D.; "der Anfang aber dieses Buches war im Kloster des Mōr Barṣaumō und seine Beendigung im Kloster des Abū Ġālib.” Nach einem zweiten Vermerk wurde ein weiterer Teil abgeschlossen im Jahre 1889 A. Gr. (1578/79 A. D.) durch Yānīs (= Johannes), Sohn des Jerusalempilgers (maqdsōyo ) Mardiros, Sohn des Jerusalempilgers Barșaumō aus dem Dorf Wank. ${ }^{46}$ Der Name Mardiros (das ist die westarmenische Aussprache von Martyrios) spricht für armenische Abstammung. Die Hs. Oxford Marsh 706 (= Syr. 15) bestätigt das für den Schreiber ausdrücklich (bar

${ }^{43}$ Monuments chrétiens, 192, Fußnote 27.

${ }^{44}$ Dolabany, Catalogue of Syriac Manuscripts, 7-9.

45 So Arthur Vööbus, Syrische Kanonessammlungen. Ein Beitrag zur Quellenkunde. I. Westsyrische Originalurkunden (Löwen, 1970) (= CSCO 307, 317) 236. In seinem Aufsatz "Die Entdeckung einer neuen Schrift des Mōšè bar Kēphā über das Priestertum” (in: Ostkirchliche Studien 23 [1974]: 324-7) verweist er auf S. 325 nur auf "eine kleine Notiz am Ende, wonach die Handschrift im Monat 'Āb im Jahre 1885 A. Gr., d. h. im August 1574 n. Chr. fertiggestellt wurde. Als Ort ihrer Herstellung wird das Kloster des Mōr Barșaumō genannt.” Das entspricht der Angabe bei Dolabani.

46 Isaac Armalet, Catalogue des manuscrits de Charfet [arabisch] Jounieh 1936, $92 \mathrm{f}$. 
maqdšōyō Barșaumō armnōyō). ${ }^{47}$ Bei der Datierung wird neben dem Patriarchen, dem Maphrian und dem Metropoliten von Jerusalem der heimische Bischof von Gargar genannt.

h. Der nächste Beleg ist die Hs. Leningrad 13, ein Tetraevangelium. Sie wurde beendet "am 9. September 1889 A. Gr. [= 1578 A. D.] ...; geschrieben wurde sie im heiligen Kloster des Mōr Barșaumō ..." Bei der Datierung werden dieselben kirchlichen Würdenträger genannt. Der Schreiber, der Priestermönch Iwannīs, bittet dann um ein Gebet für "Rabban Hananyā, den Abt des Klosters des Mōr Barṣaumō, der mein Meister und mein Lehrer ist." Der Kopist stammt eigentlich nicht aus dem BarșaumōKloster, denn am Anfang des Lukasevangeliums bezeichnet er sich als 'Yō'annīs, Mönch aus dem Kloster der Muttergottes Maria und des seligen Mōr Zakai, das auf dem nördlichen Berg oberhalb des gesegneten Kastra Wank, bekannt als Kloster des Abū Gālib, und (oberhalb von) Tabsiyas ist, im Gebiet von Gargar." 48 Der Wechsel eines Mönches von einem Kloster zum anderen war nicht unüblich. Trotz der unterschiedlichen Schreibung des Namens Johannes dürfte der Kopist der beiden Teile der Hs. Scharfeh 5/3 und derjenige der Hs. Leningrad 13 also identisch sein. Hananyā und sein Schüler Johannes sind sicherlich dieselben Mönche, die schon 1568/9 nach der Hs. Aleppo Orth. 116 (s. oben d) dort wohnten. Da der Schreiber der Handschrift Aleppo Orth 116 zum Gebet für die Mönche von Mōr Abhai und Mōr Barșaumō auffordert (aber nicht für die von Abū Gālib), hat es offenbar engere Beziehungen zwischen diesen beiden Klöstern gegeben. Vielleicht wurde das Barșaumō-Kloster damals von Mōr Abhai aus wieder besiedelt. Ob 1578 mehr als zwei Mönche dort lebten, wissen wir nicht.

47 Robert Payne Smith, Catalogi codicum manuscriptorum bibliotheca Bodleianae, pars sexta, codices syriacos, carshunicos, Mendaeos complectens (Oxford, 1864) 56-8. Vgl. auch Baraum, Histoire, 460.

${ }^{48}$ Beschreibung der Handschrift: I. W. Pigulewskaja, Katalog sirijskich rukopisej Leningrada (Moskau/Leningrad, 1960. = Palestinskij Sbornik 69) 45-8. 
i. Aus derselben Zeit stammt die Handschrift Dublin 1503. Sie wurde 1578 A. D. ebenfalls von Johannes, Sohn des Mardiros, Sohn des (Bar)ṣaumō, im Kloster des Mōr Barșaumō kopiert. ${ }^{49}$

j. Nach der in der Hs. Oxford Hunt. 444 (= Syr. 68) enthaltenen Ordinationsliste wurden "am Gedächtnistag der Apostel des Jahres 1891 A. Gr.” (1580 A. D.) Michael und Gīwargīs zu Diakonen "für das Kloster des heiligen Mōr Barșaumō" geweiht (fol. 2b). Daß es Mönche waren, läßt sich nicht sicher sagen, ist aber wahrscheinlich. Bei Michael wird es sich um den späteren Bischof Gregorios Michael von Gargar handeln, der zumindest zeitweilig im Barșaumō-Kloster residierte (s. unten $\mathrm{p}$ und $\mathrm{q}$ ), oder um den gleichnamigen Abt (s. unten q). Am 5. Dezember 1895 A. G. (1583 A. D.) erhielt der Mönch Toros (Theodoros) - der Namensform nach armenischer Abkunft die Weihe zum Priester "für das Kloster des Barșaumō" (fol. 3b). ${ }^{50}$ Die Zahl der Mönche scheint also angestiegen zu sein.

k. 1583 besuchte der päpstliche Legat Leonardo Abel das Gebiet von Gargar, um mit dem syrisch-orthodoxen Patriarchen im Kloster des Mōr Abḥai zusammenzutreffen. In seiner "Relazione" über seine Mission an Papst Sixtus V. erwähnt er daneben die Orte Gargar und Urbīš. In der Aufzählung der Bischöfe kommt ein "Anania Vescovo di Santo Barsome"51 vor, womit unser Kloster und der in der Hs. Leningrad 13 genannte frühere Abt dieses Namens (s. oben [9] und [13]); gemeint sein dürften; dieser Hananyā wurde vom Patriarchen Dawūdšāh (1576-

49 T. K. Abbott, Catalogue of the Manuscripts in the Library of Trinity College, Dublin (Dublin, 1900) 403 ("in the convent of Mār Bar-Sammā”).

${ }^{50}$ Er kann nicht mit dem Besteller der Hs. Oxford Marsh 706 (= Syr. 15) namens Theodoros identisch sein, weil dieser 1578 bereits Priester war.

51 Relazione Di quanto ha trattato il Vescovo di Sidonia nella sua missione in Oriente data alla Santità di N. Signore Sisto V a XIX Aprile MCLXXXVII, in: J. D. Mansi, Stephani Balurii Tutelensis Miscellanea novo ordine digesta et ... aucta, Band 4 (Lucca, 1776) 150-8 (hier: 153); Ag̀nāṭius Anțūn Hāyik, 'Allāqāt kanisat as-suryàn al-ya'äqiba mac al-kursì ar-rasūlì (Beirut, 1985) 129-42 (hier: 139). Unrichtig Adolphe d'Avril (Hrsg.), "Relation de l'évêque de Sidon," Revue de l'Orient Chrétien 3 (1898): 200-16; 215: "Ananias, évêque de Sainte-Bertonie." 
1581) zum Bischof von Gargar geweiht, ${ }^{52}$ residierte aber — wie üblich — weiter in einem Kloster. Honigmann ging offenbar davon aus, daß das berühmte Kloster nicht wieder bewohnt war und vermutete zu Unrecht, daß Abel ein anderes meinte: "Il s'agit probablement du couvent du saint près de Hašraï, Begadšì et Tell Qobab" bei Kephartūtā (südwestlich von Mardin). ${ }^{53}$ Zum einen spricht die Reihenfolge der Bischofssitze in der Liste

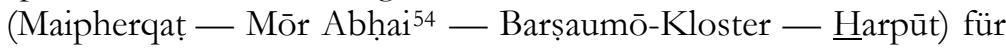
das Kloster in unserem Gebiet, zum anderen paßt der Name des Bischofs zu den sonstigen Nachrichten aus dieser Zeit. Hananyā wird nämlich — zusätzlich mit seinem Amtsnamen Athanasios für die Jahre 1587 bis 1594 in weiteren Handschriften erwähnt (s. etwa oben [13], unten [17] und [18]).

1. Am 13. Dezember 1587 A. D. (1899 A. Gr.) stellte der Priestermönch Sōhdō, Sohn des Maqdis(i) Ohannes ('whns) aus dem Gebiet von Gargar einen Liturgiekommentar, die Hs. Mardin Orth. 121, fertig, und zwar zur Zeit des Patriarchen Dawūdšāh, des Maphrians Basileios Pilatos und "unseres Bischofs Mōr Athanasios Hananyā." 55 Wegen der armenischen Form des Vatersnamens ist auch bei ihm armenische Abstammung wahrscheinlich. Am Schluß des in nicht ganz fehlerfreiem Syrisch verfaßten Kolophons heißt es: ktbt hn' ktb' dyr' bryk' d-mry Brșwm' twr' d-Psk' lwqbl țwr' d-qryr "Ich habe dieses Buch geschrieben (im?) gesegneten Kloster unseres Herrn Mōr Barșaumō (auf?) dem Berg PSK' gegenüber dem Berg von Qarīr. ${ }^{50 " 57}$

${ }^{52}$ Ms. Cambridge Dd. 81, s. William Wright and Stanley Arthur Cook, A Catalogue of the Syriac Manuscripts preserved in the Library of the University of Cambridge, vol. II (Cambridge, 1901) 985.

53 Le couvent de Barsaumā, 180, Fußnote 7.

54 Beim Kloster des Mōr Abhai gibt Abel keinen Namen eines Bischofs an; wahrscheinlich gab es dort damals keinen, weil ja der Metropolit von Gargar im Barșaumō-Kloster residierte.

${ }_{55}$ Bisher nicht katalogisiert.

56 Die Namen der Berge kommen auch in der Hs. Harpūt 34 von 1622 vor (s. unten q); weitere Belege dafür kenne ich nicht.

57 Sōhdō kopierte auch die Hss. Oxford Marsh 528 (= Syr. 199) (1594) und Damaskus Orth. 5/9 (1600), offenbar jedoch ohne Hinweis auf das Barșaumō-Kloster. Diese beiden Daten gibt Barsaum, Histoire, 495, Nr. 255, an. 
m. Am 18. Juli 1899 A. Gr. (= 1588 A. D.) beendete der uns schon bekannte Johannes, Sohn des Mardiros, die Hs. Aleppo Orth. 4,58 ein Evangeliar, zur Zeit des Patriarchen Ignatios Dawūdšāh, des Maphrians Basileios Pilatos und des Bischofs Athanasios von Gargar. Die Handschrift entstand auf Veranlassung der Gläubigen des Dorfes Tabsiyas im Gebiet von Gargar, in der Nachbarschaft des Kloster des Mōr Zakai, in dem das Buch geschrieben wurde, des Klosters des Abū Gālib, nämlich Kastra Wank, des Klosters des Mōr Barșaumō, des Hauptes der Asketen (rǐsō d-abilêe), und des Klosters des Mōr Abhaia. ${ }^{59}$

n. Da der frühere Abt Hananyā Bischof geworden war (s. oben k), wurde offenbar ein neuer ernannt. Barsaum gibt für 1590 den Mönch Barșaumō, Sohn des Astrazadūr aus Gargar als Abt des Barșaumō-Klosters an, ${ }^{60}$ dem Vatersnamen (armenisch Astuacatur $=$ Theodatus) nach ebenfalls Armenier; ich kann ihn nicht belegen. An anderer Stelle nennt Barsaum allerdings für 1590 Johannes aus Wank als Abt des Barșaumō-Klosters. ${ }^{61}$ Das wird eher stimmen, denn bei ihm dürfte es sich um den uns schon bekannten Johannes, Sohn des Mardiros, handeln, der sich vorher zeitweise im Barșaumō-Kloster aufgehalten hatte, Schüler des früheren Abtes Hananyā war (s. oben h) und dann um 1595 Bischof wurde (s. unten [20]).

o. 1594 bezeichnet Johannes, Sohn des Mardiros, in der Hs. Scharfeh Patr. 277 die Lage des Zakai-Klosters wie folgt: "im Gebiet von Gargar, auf dem Berg des Klosters des Mōr Barșaum." 62 Wir kennen noch eine Reihe weiterer Handschriften, die auf ihn zurückgehen, aber nicht Bezug auf das BarșaumōKloster nehmen. In dem Tetraevangelium Oxford Poc. 1 (= Syr. 31), geschrieben 1594/5 im Kloster des Mōr Zakai, nennt er zunächst seinen Namen Wānīs (= Johannes) bar Mardiros,

${ }^{58}$ Bisher nicht katalogisiert.

${ }^{59}$ Nach Barsaum, Histoire 461 soll die Handschrift kurze geschichtliche Nachrichten über die Klöster von Gargar enthalten.

${ }^{60}$ Histoire, 494 (No. 251).

61 Barsaum, Histoire, 460, gibt als Datum für die Weihe zum Metropoliten an: "um 1599."

${ }^{62}$ Sony, Catalogue ... de Charfet, 72f. (Nr. 223). Die Angabe "Berg des Mōr Barșaumō" (tūiō d-Mōr B.) findet sich auch in der Chronik Michaels des Syrers (Chabot, Chronique de Michel, III 163a, $290=$ IV 574, 647). 
erscheint in der Handschrift später aber als (Metropolit) "Gregorios (Johannes) von Kappadokien." ${ }^{3} \mathrm{Er}$ ist also nach 1594/5 A. D. Bischof geworden. ${ }^{64}$

p. Die Hs. Istanbul Meryam Ana 49/46 (aus dem Besitz von Thomas Başaranlar in Diyarbakir) enthält eine Sammlung von Heiligenviten. Nach der Geschichte des Barșaumō findet sich ein Vermerk vom 13. Februar 1599 A. D. (1810 A. Gr.), in dem der Schreiber Bischof Gregorios (Michael) von Gargar angibt, er habe das Buch geschrieben "in der Muttergotteskirche des gesegneten Kastra Wank, das bekannt ist als Kloster des Abū Gāalib ... in der Nähe des Klosters der Muttergottes Maria und des heiligen ... Mōr Zakai und der übrigen Klöster, die in unserer Zeit entleert sind von Menschen und auch zerstört, und ferner in der Nähe des Klosters und der Höhle des heiligen Mōr Barṣaumō." ${ }^{65}$ Die Höhle, die auch im nächsten Beleg erscheint, läßt wieder an das alte Kloster aus dem 5. Jh. denken.

q. 1622 A. D. (1933 A. Gr.) schrieb der Priestermönch Barșaum, Sohn des MWSS (wohl Mowsēs, die armenische Form des Namens Mose) einen Şhīmō für die Winterzeit (olim Hs. Harpūt 39), ${ }^{66}$ zur Zeit “... unseres Metropoliten von Gargar, Mōr Michael, im Kloster des Mōr Barșaumō.” Der Kopist schrieb "in einer Höhle im Berg PSK', gegenüber dem Berg Qarīrō, im Gebiet von Kak tāa ${ }^{67}$ in der Gegend von Melitene, im nördlichen Berg." Die Namen der Berge sind uns schon in der Hs. von 1587 (oben l). begegnet. Der Kopist gedenkt des Abtes Michael und des Mönches Isaak, eines "würdigen Greises." Da er den Abt als "Rabban" kennzeichnet, kann dieser kaum mit dem zuvor genannten "Bischof" Michael von Gargar "im Kloster des Mōr Barṣaumō"

63 Payne Smith, Catalogi, 94-6. Nach dem syrischen Text im Katalog wurde die Handschrift 1906 ('sw) A. Gr., d. h. 1594/5 A. D. geschrieben, was zu den sonstigen Daten für den Schreiber paßt. Die Übersetzung "anno Graecorum 1900 (A. D. 1695)" ist jedenfalls falsch.

${ }^{64}$ Barsaum, Histoire, 460, gibt als Datum für die Weihe zum Metropoliten an: "um 1599."

${ }^{65}$ Die Handschrift ist nicht katalogisiert. Die Angaben verdanke ich Andrew Palmer.

${ }^{66}$ Dolabany, Catalogue of Syriac Manuscripts, $71 \mathrm{f}$.

${ }^{67} \mathrm{Vgl}$. Honigmann, Le couvent de Barșaumā, 38f., 82. 
identisch sein. ${ }^{68}$ Einer von beiden ist wohl der Michael, der 1580 zum Diakon für das Kloster geweiht worden war (s. oben [15]).

Bei dem Bischof Michael handelt es sich sicherlich um Michael, Sohn des Barșaumō aus Urbīš, der mehrere Handschriften kopierte, unter anderen 1588 den erhaltenen Archetypus der Chronik Michaels des Syrers. ${ }^{69} \mathrm{Ob}$ diese Abschrift im Barșaumō-Kloster erfolgte, also am Entstehungsort der Chronik, wissen wir nicht, weil sich nicht feststellen läßt, ob Michael damals dort lebte. ${ }^{70}$ Für 1580 und 1594 ist sein Aufenthalt im Kloster des Mōr Abhai bezeugt. Leider gibt er in seinen Kolophonen selten den Ort an. Seine Abschrift der Chronik befand sich Mitte des 18. Jh. im Kloster des Mōr Abhai und diente dem Mönch Hannā ibn Isā aus Ṣadad als Vorlage für seine arabische Übersetzung. ${ }^{71}$ Um 1800 war sie vorübergehend in Dair az-Za farān. ${ }^{72}$ Dann gelangte sie zu einem unbekannten Zeitpunkt von Mōr Abhai nach Edessa, wo sie sich bis zur Auswanderung der dortigen syrischen Bevölkerung nach Aleppo im Jahre 1924 aufbewahrt wurde. Heute befindet sie sich in Aleppo. Michael war ab ungefähr 1600 Metropolit von Gargar mit dem Amtsnamen Gregorios. Mehr Mönche als die Genannten wird das Kloster damals kaum gehabt haben.

r. Nach der Ordinationsliste der Hs. Paris Syr. 395 nahm 1623/4 (?) Kyrillos Johannes aus Gargar, Metropolit von Hiṣn Ziyād (d. h. ㅂarpūt), eine Weihe für das Barșaumō-Kloster vor. ${ }^{73}$

${ }^{68}$ Barsaum, Histoire, 494 (Nr. 251) kennt einen "Rabbān Mīhāa ibn Nağğāar Daulatšāh al-Wankī al-Karkarī (1589-1606)."

${ }^{69}$ J.-B. Chabot, Chronique de Michel le Syrien, I (Paris, 1899) xxxvi f.

${ }_{70}$ Die Angabe von Honigmann, Le couvent de Barșaumā, 82, Fußnote 1, die Handschrift sei in Urbǐš geschrieben, läßt sich der von ihm zitierten Stelle nicht entnehmen. Der Schreiber stammte nur aus Urbīš.

71 Athanasius Afram Baraum, Nubda min ta'rih al-'abrašiyyāt assuryāniyya, in: al-Mağallat al-batriyarkiya 7 (1940): 187. Vgl. auch Georg Graf, Geschichte der christlichen arabischen Literatur, Band 2 (Vatikanstadt, 1947) 267.

72 Chabot, Chronique de Michel, I, xxxviii.

${ }^{73}$ Françoise Briquel-Chatonnet, Manuscrits syriaques de la Bibliothèque nationale de France ... (Paris, 1997) 115, die leider die Weihen nicht einzeln aufführt, so daß nicht sicher ist, wann die Weihe für das BarșaumōKloster (fol. 13) stattfand. Die Hs. war früher in Istanbul, s. Dolabani, 
s. 1623/4 (1935 A. Gr.) weiht vermutlich derselbe Bischof Kyrillos (Johannes) jemanden für das Barșaumō-Kloster, wie der Weiheliste der Handschrift Scharfeh 111 zu entnehmen ist. ${ }^{74}$

t. Die Ordinationsliste der Hs. Damaskus, Syrisch-orthodoxes Patriarchat 5/16 enthält die Weiheeinträge des für dieses Gebiet zuständigen Metropoliten Kyrillos Ephräm von Gargar ${ }^{75}$ für die Jahre 1638 bis 1655. Die Weihen finden — soweit angegeben im Zakai-Kloster (1638), in Wank/Abū Gālib (1645, 1654), im Barșaumō-Kloster "im gesegneten Gebiet von Kaḳtā” (1645, 1647), im Abhai-Kloster (1647, 1654), in Urbī̌s (1654) und Karmō dDakyō (1655) statt. 1645 und 1647 wird jeweils ein Barșaumō im Barṣaumō-Kloster (und wohl für das Kloster) zum Diakon geweiht.

u. Von 1658 bis 1661 entstand in diesem Gebiet auch die Hs. Mardin Orth. 326, die den Nomokanon des Barhebraeus und einige weitere Rechtstexte enthält. ${ }^{76}$ In ihr finden sich mehrere aufschlußreiche Schreibervermerke. Kopist war der Metropolit Kyrillos Ephräm von Gargar, der uns im vorigen Abschnitt schon begegnet ist. Er taucht auch in Schreibervermerken weiterer Handschriften auf. Sein Vater hieß 'Ohannes, was wieder auf armenische Abstammung deutet. Geboren ist er kurz nach 1580, weil er 1659 in der Hs. Harvard Syr. 54 angibt, er sei 77 Jahre alt. ${ }^{77}$

Catalogue of Syriac Manuscripts, 10-3 (mit Weiheliste, in der aber gerade der Eintrag für das Barșaumō-Kloster fehlt).

${ }^{74}$ Sony, Catalogue ... de Charfet, 285b (No. 780). Leider ist im Katalog auch diese Liste zu pauschal aufgenommen.

${ }^{75}$ Er fehlt bei Fiey, Pour un Oriens Christianus Novus, 200.

${ }^{76} \mathrm{~S}$. Arthur Vööbus, An Unknown Recension of the Syro-Roman Lawbook (Stockholm, 1977) 7f (as No. 316), und mehr oder weniger gleich in zahlreichen weiteren Veröffentlichungen. Ein Katalog der Handschriften juristischen Inhalts von Hubert Kaufhold und Walter Selb ist in Vorbereitung.

77 D. S. Margoliouth, "The Syro-Armenian Dialect," The Journal of the Royal Asiatic Society (1898): 839-61 (insbesondere: 840, 846); M. H. GoshenGottstein, Syriac Manuscripts in the Harvard College Library. A Catalogue (Missoula, 1979) 59f. Vgl. auch Barsaum, Histoire, 34 (Handschrift in Boston). Entgegen der Annahme von Vincenzo Poggi und Mar Grigorios, "Hannā Ibrāhīm, Il commento al Trisagio di Giovanni Bar Qūrsūs," Orientalia Christiana Periodica 52 (1986): 202-10 (hier: 207) kann Ephräm nicht erst 1612 geboren sein. Die Angabe beruht auf Barsaum, Histoire, 495, der für Ephräm die Jahreszahlen 1612-75 angibt, damit nach meinen 
1622 schrieb er den größten Teil der Hs. Vat. Syr. 159, die verschiedene theologische Schriften enthält; einen weiteren Teil davon kopierte er 1629 bereits als Metropolit von Hattāk $\underline{a},{ }^{78}$ einem Bischofssitz in der Nähe von Maipherqaț. ${ }^{79} 1638$ wechselte er auf Wunsch des Patriarchen von Hattākāa auf den Sitz von Gargar über; der in Urbīš (Mōr Abhai) residierende Bischof (Gregorios) Pilatos ging später nach Edessa.80 Trotz seines Wechsels nach Gargar bezeichnet Ephräm in einer 1950 A. Gr. (1638/39 A. Gr.) beendeten Handschrift (Scharfeh Patr. 221) Pilatos noch als Ortsbischof: "zur Zeit ... des Mōr Gregorios Pilatos aus Kastra Urbīšs, des Metropoliten von Gargar und Mōr Abhai." ${ }^{11}$ Wie die Lage kirchenrechtlich war, wird nicht klar.

Offenbar für seine Amtstätigkeit als Bischof fertigte er im Kloster des Mōr Zakai das Pontificale Damaskus Orth. 5/16 an, das er 1639 A. D. (1950 A. Gr.) beendete, ${ }^{82}$ aber vorher begonnen haben muß, weil sich bereits die oben unter [25]). erwähnten Weiheeinträge ab 1638 darin befinden. Die letzte mir bekannte

Erfahrungen aber nicht unbedingt die Lebensdaten meint, sondern den ersten und letzten Beleg, den er für die betreffende Person kennt.

78 St. E. und J. S. Assemani, Bibliothecae Apostolicae Vaticanae codicum manuscriptorum catalogus, partis primae tomus tertius (Rom, 1759) 307-19, insbesondere 312f., 316. Vgl. auch Assemani, Bibliotheca Orientalis, II, $237 f$. und Poggi und Mar Grigorios, "Hannā Ibrāhīm, Il commento," 207 (deren Angabe, er habe die Handschrift zwischen 1628 und 1640 kopiert, muß ein Versehen sein; der spätere Teil wurde im September 1940. A. Gr., also 1629 A. D. beendet).

${ }^{79} \mathrm{Zu}$ Hattākāa vgl. Honigmann, Le couvent de Barșaumā, 166.

80 Hs. Harpūt Nr. 39, s. Dolabany, Catalogue of Syriac Manuscripts in Syrian churches and Monasteries, 73. Fiey, Pour un Oriens Christianus Novus, 209 führt ihn unter den Bischöfen von Hattākāa auf, ohne nähere Daten anzugeben; er vermerkt nur, offenbar in Anschluß an die Literaturgeschichte von Patriarch Barsaum (S. 495, Nr. 263), daß er als Kopist von 1612 bis 1675 bekannt sei. Der Name ist bei Fiey unrichtig angegeben. "Jean" ist nicht sein Bischofsname, sondern der Name des Vaters (Fiey verkennt die armenische Namensform und schreibt "Ahanis"). Barsaum bezeichnet ihn an der angegebenen Stelle nur als Bischof von Hattākāà.

81 Sony, Catalogue ... de Charfet, 339 (No. 856).

82 Yuhanon Dulabani, René Lavenant, Sebastian Brock, Samir Khalil Samir, "Catalogue des manuscrits de la bibliothèque du patriarcat Syrien Orthodoxe à Homs (auj. à Damas)," Parole de l'Orient 19 (1994): 555-661 (here: 577). 
Handschrift aus den Jahren 1658 bis 1661, Mardin Orth. 316, soll uns gleich noch näher beschäftigen. Barsaum vermerkt als letztes Datum 1675. Der Bischof müßte dann über 90 Jahre alt geworden sein.

In einem Kolophon von 1660/61 in der Mardiner Handschrift gibt Ephräm an, daß es in dem Jahr im Gebiet von Gargar eine große Hungersnot gegeben habe, so daß der Preis für das Getreide sehr hoch gewesen sei; außerdem sei der Euphrat so gestiegen, daß er beim Kloster des Mar Abhai die Leitern überflutet habe. Einen weiteren Vermerk, in dem unser Barșaumō-Kloster vorkommt, beendete er in der Nacht des Pfingstsonntags 1661 in der Marienkirche in Wank, zur Zeit des Patriarchen Ješū Qamšah, des Maphrians Basileios Abd al-Masịh sowie "des Bischofs von Kappadokien, der im Kloster des Mōr Abḥai wohnt, (nämlich) Mōr Gregorios Barșaumō aus Wank, und des Timotheos Abd al-Ğalīl, des Metropoliten von Amid, aus Mosul, und eines anderen Bischofs, der im Kloster unseres Herrn Mōr Barșaumō wohnt, nämlich 'Abdallāh aus Wank." Dann fährt er fort: "Gott möge Rechenschaft fordern von diesen Patriarchen am Tage des Gerichts! Im Gebiet von Gargar gibt es drei Bischöfe. Seht ihr denn, o unsere Väter, überhaupt nicht, was geschieht? Wir sehen nicht, daß Gott diese Patriarchen verflucht, die unter uns diese Trennungen verursacht haben." Er berichtet dann noch, daß er im Kloster des Mōr Zakai von Räubern überfallen und beraubt worden sei, ohne daß ihm jemand geholfen hätte; er überlege, ob er in die Stadt Edessa gehen solle. Ob er dies wahrgemacht hat, wissen wir nicht. 83

Es war damals offenbar eine Zeit innerkirchlicher Wirren. Patriarch Yešù Qamšah war 1652 zum Gegenpatriarchen geweiht worden und wurde erst 1660 nach dem Tod des seit 1639 amtierenden Patriarchen Simon allgemein anerkannt, starb aber schon 1662. Ab 1662 gab es dann den unierten Gegenpatriarchen Andreas Ahiğāan. Auf den im dritten Vermerk erwähnten Bischof von Kappadokien Gregorios Barșaumō aus Wank kommen wir gleich noch zurück (unten w). Bischof Abdallāh aus Wank (bei Gargar) war wohl derjenige, der 1653 als Rabban Abdallāh al-

83 Vermutlich sind die Kolophone der Hs. Mardin 316 die "Autobiographie," von der Patriarch Barsaum, Histoire, 137 (syr. Übersetzung: 188), spricht. Danach Poggi und Mar Grigorios, " Hannā Ibrāhīm, Il commento," 207. 
Gargarī die Hs Oxford 68 kaufte; mehr wissen wir von ihm nicht. Mit seiner Klage, daß es im Gebiet von Gargar drei Bischöfe gebe, meint Ephräm anscheinend sich selbst im Kloster des Mōr Zakai, Gregorios Barṣaumō im Kloster des Mōr Abhai und Abdallāh im Barșaumō-Kloster. Nach dem insoweit etwas distanziert klingenden Vermerk scheint der Bischof im Barșaumō-Kloster dem Gegenpatriarchen angehangen zu haben.

v. 1675/6 A. D. (1987 A. Gr.) fand eine nicht näher bezeichnete Weihe eines Rabban Barșaum, also eines Mönchs, für "Mōr Barșaumō" statt (Weiheliste der Hs. Oxford Hunt. 444 [= Syr. 68], fol. 184a). Die Eintragung wirkt allerdings ziemlich unbeholfen. Da vorher auf fol. 183a Weihen des Jahres 1673/4 für die Muttergotteskirche in Wank verzeichnet sind, ist sicher unser Barṣaumō-Kloster gemeint.

w. Am 26. August 1987 A. Gr. (1676 A. D.) beendet Rabban Jakob aus der Familie Sabadiar aus Wank die Hs. Scharfeh 32 "im Kloster des Barṣaumō, das Kloster der Stufen genannt wird." 84 Mit dem "Stufenkloster" ist sonst immer nur das Kloster des Mōr Abḥai gemeint, nicht das des Mōr Barșaumō. Ob hier ein Versehen des Kopisten oder des Katalogverfassers vorliegt, kann ich nicht feststellen. Der weitere Inhalt des Kolophons sei gleichwohl mitgeteilt, weil er für die damalige traurige Lage bezeichnend ist, die schon Bischof Kyrillos Ephräm beklagte. Das Kloster sei in gutem Zustand gewesen, "aber der Teufel war neidisch und warf Zwietracht unter die Mönche;" sie hätten den Metropoliten (Gregorios) Barșaumō aus Wank bekämpft, der sei abgereist und habe sich anderswo niedergelassen; einem Aufstand der Kurden hätten sie nicht standgehalten. Diese pessimistisch gestimmte Notiz ist die letzte mir bekannte Erwähnung des Barșaumō-Klosters. ${ }^{85}$ Seine Geschichte ging in diesen Jahren wohl endgültig zuende.

${ }^{84}$ Sony, Catalogue ... de Charfet, 75 (No. 229).

85 Nicht hierher gehört die Hs. Scharfeh Patr. 297, ein Fanqītō. Sie wurde 1722 A. D. (= 2033 A. Gr.) geschrieben "im Kloster des Mōr Theodoros, und ihre Beendigung geschah im Kloster des heiligen Mōr Barșaumō" (Sony, Catalogue ... de Charfet, 3, Nr. 112). Der Schreiber, der Diakon Joseph Ibrāhīm, stammte aus dem Dorf Șadad im Bezirk Qārā in Syrien, so daß man an die Theodorkirche in Șadad denken muß, auch wenn meines Wissens nicht belegt ist, daß sich dort ein Kloster befand. Jedenfalls ist nicht unser Barșaumō-Kloster gemeint, wie sich aus einer 


\section{III}

[29] Das Gebiet von Gargar war in den letzten Jahrhunderten sicherlich nicht ohne Bedeutung für die syrisch-orthodoxe Kirche, und seine Geschichte bedürfte einer eingehenderen Untersuchung. Die oben erwähnten Bischöfe Gregorios Johannes, Sohn des Mardiros, ${ }^{86}$ und Kyrillos Ephräm von Gargar waren von ihren geistigen Interessen her für ihre Zeit herausragende Erscheinungen. Allerdings ist der allgemeine Niedergang des kirchlichen Lebens offensichtlich. An den Weihelisten läßt sich ablesen, daß es im Bistum Gargar nur noch wenige Gemeinden gab. Wenn George Percy Badger um die Mitte des vorigen Jahrhunderts berichtet, ein syrisch-orthodoxer Metropolit habe ihm erzählt, in der Gegend von Gargar lägen nicht weniger als 60 christliche Dörfer, ${ }^{87}$ erscheint das kaum glaubhaft. Jedenfalls gab es offenbar nur in den oben genannten wenigen Ortschaften Kirchen der Syrer. Die Zahl der Mönche in den drei noch bewohnten Klöstern war offenbar nicht groß. Unter ihnen spielte das Barșaumō-Kloster die geringste Rolle. Ob in den späten Quellen das Kloster auf dem Berg gemeint ist oder das offenbar an dessen Fuß gelegene alte Kloster des heiligen Barșaumō, ist meist nicht zu entscheiden. Archäologische Untersuchungen könnten vielleicht weiterführen. Im Barșaumō-Kloster haben nach der Wiederbelebung wohl nur für etwa zweihundert Jahre Mönche gelebt, und es scheint Ende des 17. Jh. endgültig aufgegeben worden zu sein. Die Klöster des Mōr Abhai und des Abū Galīib bestanden noch einige Zeit länger. Ungünstig wirkten sich nicht nur die unsichere politische Lage, sondern auch die Streitigkeiten innerhalb der westsyrischen Kirche aus.

weiteren Handschrift desselben Kopisten ergib, nämlich einem unkatalogisierten Evangelienkommentar in der syrisch-orthodoxen Metropolie in Homs, der am 1. 4. 2032 A. Gr. (1721 A. D.) "im Kloster des Mōr Barșaumō in Șadad" beendet wurde. Weitere Nachweise für dieses Kloster kenne ich nicht. Es erscheint nicht bei H. al-Arab, "Ṣadad fi't-tā'rīh," The Patriarchal Journal, Jahrgänge 20-2 (1982-4), in Fortsetzungen. Zu weiteren Barșaumō-Klöstern vgl. Honigmann, Le couvent de Barșaumā, $44 \mathrm{f}$.

${ }^{86} \mathrm{Zu}$ ihm s. auch Macuch, Geschichte der spät- und neusyrischen Literatur, 23.

87 The Nestorians and their Rituals, vol. I (London, 1852) 318. 


\section{LITERATURVERZEICHNIS}

Abbeloos, Joannes Baptista, und Thomas Josephus Lamy, Gregorii Barhebraei chronicon ecclesiasticum, tomus I. Löwen, 1872

Abbott, T. K. Catalogue of the Manuscripts in the Library of Trinity College, Dublin. Dublin, 1900.

Anonymi auctoris chronicon ad A.C. 1234 pertinens. II. Traduit par Albert Abouna, introduction, notes et index de J. M. Fiey. CSCO, 154. Louvain 1974.

Ainsworth, W. F. Travels and Researches in Asia Minor, Mesopotamia, Chaldea and Armenia. London, 1842.

al-Arab, H. "Ṣadad fi't-tā'rịh." The Patriarchal Journal, Jahrgänge 20-2 (1982-4)

Armalet, Isaac. Catalogue des manuscrits de Charfet [arabisch]. Jounieh, 1936

Assemani, Stephanus Evodius, und Joseph Simonius. Bibliothecae Apostolicae Vaticanae codicum manuscriptorum catalogus, partis primae tomus secundus, Rom, 1758; partis primae tomus tertius, Rom, 1759

Assemani, Joseph Simonius. Bibliotheca Orientalis Clementino-Vaticana, Band 2. Rom, 1721

d'Avril, Adolphe, Hrsg. "Relation de l'évêque de Sidon." Revue de l'Orient Chrétien, 3 (1898): 200-16

Badger, George Percy. The Nestorians and their Rituals, vol. I. London, 1852.

Barsaum, Ignatios Aphrem. Histoire des sciences et de la littératur syriaque [arabisch], 2. Auflage. Aleppo, 1956 (mehrere Nachdrucke), 509. Syrische Übersetzung von Ph. Y. Dolabani: Ktōbō da-brullè bdìrè. Qāmišlīi, 1967

Barsaum, Agnatius Afram. "Nuḳba min ta'rih al-abrāšiyāt as-suryānìya." al-Mağallat al-batriyarkìya, 7 (1940).

Briquel-Chatonnet, Françoise. Manuscrits syriaques de la Bibliothèque nationale de France ... Paris, 1997

Chabot, Jean-Baptiste, Hrsg. Anonymi auctoris cbronicon ad A.C. 1234 pertinens, II. Löwen, 1916.

. Chronique de Michel le Syrien, tome I-IV. Paris, 1899-1900.

Dolabani, Yohannan. Die Patriarchen der syrisch-orthodoxen Kirche von Antiocbien [syrisch]. Holland [Glane/Losser]. 1990.

Dolabany, Filoksinos Yohanna. Catalogue of Syriac Manuscripts in St. Mark's Monastery. Ed. by Gregorios Yohanna Ibrahim. Aleppo, 1994; Catalogue of Syriac Manuscripts in Syriac Churches and Monasteries. Aleppo, 1994.

Dolabani, Yuhanna, René Lavenant, Sebastian Brock, Samir Khalil Samir. "Catalogue des manuscrits de la bibliothèque du patriarcat Syrien Orthodoxe à Homs (auj. à Damas).” Parole de l'Orient, 19 (1994): 555-661.

Fiex, Jean-Maurice. Pour un Oriens Christianus Novus. Beirut, 1993. 
Goshen-Gottstein, M. H. Syriac Manuscripts in the Harvard College Library. A Catalogue. Missoula, 1979.

Graf, Georg. Geschichte der christlichen arabischen Literatur, Band 2. Vatikanstadt, 1947.

Hayik, Ag̣nāṭius Anțūn. 'Allaqāt kanìsat as-suryān al-yàāiba mà al-kursī arrasülì. Beirut, 1985.

Hellenkemper, Hansgerd. Burgen der Kreuzritterzeit in der Grafschaft Edessa und im Königreich Kleinarmenien. Bonn, 1976.

Honigmann, Ernest. Le couvent de Barșaumà et le patriarcat jacobite d'Antioche et de Syrie. CSCO, 146. Löwen, 1954.

Humann, K., O. Puchstein. Reisen in Kleinasien und Syrien. Berlin, 1890.

Kawerau, Peter. Die jakobitische Kirche im Zeitalter der syrischen Renaissance. Berlin, 1960.

van Lantschoot, Arn. Inventaire des manuscrits syriaques des Fonds Vatican (490-631) Barberini Oriental et Neofiti. Vatikanstadt, 1965.

Macler, Frédéric. Rapport sur une mission scientifique en Belgique. Hollande, Danemark et Suède, Paris, 1924.

Macuch, Rudolf. Geschichte der spät- und neusyrischen Literatur. Berlin/New York, 1976.

Mansi, J.D. Stephani Baluqii Tutelensis Miscellanea novo ordine digesta et ... aucta, Band 4. Lucca, 1776.

Margoliouth, D.S. "The Syro-Armenian Dialect." The Journal of the Royal Asiatic Society (1898): 839-61.

Mounayer, Joseph. Les synodes syriens Jacobites. Beirut, 1964.

Nau, François. "Résumé des monographies syriaques: Barsauma ..." Revue de l'Orient Chrétien, 18 (1913): 270-6, 379-89; 19 (1914): 113-34, 278-89.

Pigulewskaja, I. W. Katalog sirijskich rukopisej Leningrada. Moskau/ Leningrad, 1960 (= Palestinskij Sbornik, 69).

Poggi, Vincenzo, Mar Grigorios, Hanna Ibrāhīm, "Il commento al Trisagio di Giovanni Bar Qūrsūs.” Orientalia Christiana Periodica, 52 (1986): 202-10

Pognon, Henri. Inscriptions sémitiques de la Syrie, de la Mésopotamie et de la région de Mossoul. Paris, 1907.

Smith, Robert Payne. Catalogi codicum manuscriptorum bibliotheca Bodleianae, pars sexta, codices syriacos, carshunicos, Mendaeos complectens. Oxford, 1864.

Sony, Behnam. Le Catalogue des manuscrits du patriarcat au couvent de CharfetLiban [arabisch]. Beirut, 1993.

Thierry, M. "Monuments chrétiens inédits de Haute-Mésopotamie." Syria, 70 (1993): 179-204

Vööbus, Arthur. History of Asceticism in the Syrian Orient, Band 2. Löwen, 1960. 
Vööbus, Arthur. Handschriftliche Überlieferung der Memre-Dichtung des Ja'qob von Serug, III. CSCO, 421. Löwen, 1980.

- Syrische Kanonessammlungen. Ein Beitrag zur Quellenkunde. I. Westsyrische Originalurkunden. CSCO, 307, 317. Louvain, 1970.

"Die Entdeckung einer neuen Schrift des Mōšē bar Kēphā über das Priestertum." Ostkircbliche Studien, 23 (1974): 324-7.

- An Unknown Recension of the Syro-Roman Lawbook. Stockholm, 1977.

Wright, William. Catalogue of Syriac Manuscripts in the British Museum, part 1. London, 1870.

Wright, William, and Stanley Arthur Cook. A Catalogue of the Syriac Manuscripts preserved in the Library of the University of Cambridge, vol. II. Cambridge, 1901. 